\title{
Engineering crops of the future: CRISPR approaches to develop climate-resilient and disease-resistant plants
}

\author{
Syed Shan-e-Ali Zaidi ${ }^{1}$, Ahmed Mahas ${ }^{2}$, Hervé Vanderschuren ${ }^{1,3}$ and Magdy M. Mahfouz ${ }^{2^{*}}$ (D)
}

\author{
* Correspondence: magdy. \\ mahfouz@kaust.edu.sa \\ 2Laboratory for Genome \\ Engineering and Synthetic Biology, \\ Division of Biological Sciences, 4700 \\ King Abdullah University of Science \\ and Technology, Thuwal \\ 23955-6900, Saudi Arabia \\ Full list of author information is \\ available at the end of the article
}

\begin{abstract}
To meet increasing global food demand, breeders and scientists aim to improve the yield and quality of major food crops. Plant diseases threaten food security and are expected to increase because of climate change. CRISPR genome-editing technology opens new opportunities to engineer disease resistance traits. With precise genome engineering and transgene-free applications, CRISPR is expected to resolve the major challenges to crop improvement. Here, we discuss the latest developments in CRISPR technologies for engineering resistance to viruses, bacteria, fungi, and pests. We conclude by highlighting current concerns and gaps in technology, as well as outstanding questions for future research.
\end{abstract}

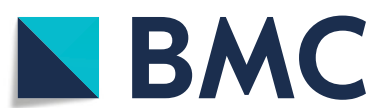

(c) The Author(s). 2020 Open Access This article is licensed under a Creative Commons Attribution 4.0 International License, which permits use, sharing, adaptation, distribution and reproduction in any medium or format, as long as you give appropriate credit to the original author(s) and the source, provide a link to the Creative Commons licence, and indicate if changes were made. The images or other third party material in this article are included in the article's Creative Commons licence, unless indicated otherwise in a credit line to the material. If material is not included in the article's Creative Commons licence and your intended use is not permitted by statutory regulation or exceeds the permitted use, you will need to obtain permission directly from the copyright holder. To view a copy of this licence, visit http://creativecommons.org/licenses/by/4.0/. The Creative Commons Public Domain Dedication waiver (http://creativecommons.org/publicdomain/zero/1.0/) applies to the data made available in this article, unless otherwise stated in a credit line to the data. 
to relevant abiotic stresses such as drought, salinity, and flooding, and biotic stresses such as insects and pathogens. As a result, the average crop yield per hectare of agricultural land has increased significantly since the Green Revolution of the 1950s and 1960s [3, 7]. The developing world has witnessed an extraordinary period of growth in food crop productivity during the past few decades, despite increasing land scarcity and rising land values. While populations more than doubled, cereal crop production tripled during this period, with only a 30\% increase in the area of land under cultivation. This pattern underscores the importance of improved crop varieties in meeting rising food demands in the past. It must be noted here that the productivity boost during the Green Revolution was due not only to improved crop varieties but also to strong investment in crop research, infrastructure, and market development and appropriate policy support, highlighting the importance of these processes for the future [7]. Nevertheless, it can be concluded that improved food crop varieties will make it possible to address the challenges of food scarcity and mitigate the effect of climate change on agriculture.

\section{Production of engineered crops via new plant breeding technologies}

Crop varieties have conventionally been developed by farmers and crop breeders using basic techniques such as the selection of plants with desirable characteristic for propagation. Modern plant breeding techniques added marker-assisted selection and genetic modification to the crop improvement toolkit. These methods have been reviewed elsewhere $[8,9]$. Briefly, a genetically modified (GM) crop variety is developed by (1) identification of a piece of DNA that confers the trait of interest, for example, a gene responsible for virus resistance; (2) cloning of the DNA into the carrier or vector plasmid; (3) delivery of the DNA to the target plant; and (4) generation of modified plants with the desired trait, e.g., virus resistance. GM crop production has been controversial mainly because of fear-based agricultural policies driven by limited public understanding, ineffective information sharing by scientists, and inaccurate portrayals by NGOs and anti-GM lobbyists [10]. Apart from social and economic concerns such as ownership, stewardship, product regulation, and market development, one major concern related to GM crops is the extensive use of certain agrochemicals (such as glyphosate) in conjunction with herbicide-tolerant GM crop varieties and the retention of antibiotic-resistance genes from the production pipeline in the GM variety. These concerns have led to the enactment of strict regulations for GM crops, which not only make the end products expensive but also slow the delivery of new varieties to farmers, making it more difficult for breeders to produce varieties suited to current threats to crops.

While society remains divided over the use of GM crops, new plant breeding technologies (NPBTs) have recently emerged as alternative approaches to speed up the introduction of improved traits. NPBTs include precision genome-modification platforms such as the clustered regularly interspaced short palindromic repeat (CRISPR)/ CRISPR-associated protein (Cas) and transcription activator-like effector nuclease (TALEN) methodologies [11]. In addition to genome editing, NPBTs include technological advances that shorten the breeding cycles and accelerate crop research, such as speed breeding [12, 13] and next-generation genotyping [14] and phenotyping platforms [15]. For some important crops that have a flowering behavior difficult for 
breeding (such as cassava, Manihot esculenta) or are sterile (such as banana, Musa acuminata), genome editing provides an efficient and robust breeding approach, given the alternative breeding approaches are either significantly inefficient or not applicable [16].

Genome-editing NPBTs differ from conventional GM methods in various ways. For example, TALENs and CRISPR-Cas can be used for precise genetic manipulation without introducing exogenous DNA such as antibiotic-resistant genes, thus eliminating the fear that foreign DNA may be present in the final product [17]. Whereas classical GM crop production requires the insertion of foreign DNA (transfer DNA, or T-DNA, from Agrobacterium species), some genome-editing protocols do not require T-DNA insertion, such as CRISPR via a ribonucleoprotein (RNP) complex or via virus-based DNA replicons to induce precisely targeted edits in the crop plant DNA [18-20]. Further elaborations of this methodology, including protoplast delivery of preassembled CRIS PR-Cas RNPs, transient expression of programmable nucleases through agroinfiltration, and site-specific integration of a CRISPR array in other chromosomal locations followed by removal via segregation, have paved the way toward developing transgenefree CRISPR plants [17, 21, 22].

These recent developments in NPBTs make it possible for new food products to reach the market quickly at affordable prices. Recent examples of such products include browning-resistant mushrooms [23], high-amylopectin waxy corn (Zea mays) [24], and false flax (Camelina sativa) with enhanced omega-3 oil [25] -all of which were developed using CRISPR and approved by the US Department of Agriculture (USDA) in record time. In summary, the latest developments in NPBTs make them excellent tools with which to produce the crops of the future, by making it possible to address concerns related to GM crops and because of their precision, robustness, and timely regulation.

\section{CRISPR-mediated genome editing: the evolution of site-specific nucleases}

Genome editing uses site-specific nucleases (SSNs), which can be designed to bind and cleave a specific nucleic acid sequence, introducing double-stranded breaks (DSBs) at or near the target site [26]. There are four major classes of SSNs: meganucleases, zincfinger nucleases (ZFNs), TALENs, and Cas proteins [26, 27]. These SSNs have significant potential for plant breeding, as they provide multifaceted mechanisms to modulate host genome structure and function, including gene knock-out, knock-in, and stacking, targeted mutagenesis, and modulation of translation. SSNs offer significant economic advantages and save time compared to conventional plant breeding approaches, which can take up to 10 years for variety development [28].

Notably, the CRISPR/Cas system has emerged as the leading, ground-breaking SSN and, although its utility for plant genome editing was first demonstrated only in 2013 [29-31], its applications in plants have increased rapidly compared to other NPBTs. Research using CRISPR has introduced important agricultural traits including heat, cold, and herbicide tolerance; viral, bacterial, and fungal resistance; and increased grain size and weight into many economically important crops, such as rice (Oryza sativa), wheat (Triticum aestivum), maize (Z. mays), tomato (Solanum lycopersicum), potato (Solanum tuberosum), tobacco (Nicotiana tabacum), cotton (Gossypium spp.), soybean (Glycine max), and brassicas [28]. Importantly, several groups have recently accomplished those genome alterations using transgene-free systems. 
The working principle of CRISPR-Cas9 has been well explained in several recent reviews $[28,32]$. Briefly, CRISPR-Cas9 is a type II adaptive immune system, identified initially in Streptococcus pyogenes, that provides prokaryotes with defenses against invading phages [33]. This system, the simplest CRISPR type, relies on the induction of site-specific DSBs in the DNA of the invading virus. These DSBs consequently induce a cellular DNA-repair mechanism either via non-homologous end-joining (NHEJ), which leads to imprecise repair, or via homology-directed repair (HDR), which leads to precise repair. Repair introduces insertions or deletions (indels) in the invader virus DNA and leads to a dysfunctional virus, providing natural defense to the bacteria against viruses.

In an engineered system, a CRISPR locus transcribes a short CRISPR RNA (crRNA) that hybridizes to a complementary sequence on the targeted genome (protospacer) adjacent to the protospacer-associated motif site (PAM). In the case of S. pyogenes, the PAM is a trinucleotide sequence, canonically $5^{\prime}$-NGG-3', that is essential for Cas9 to selectively recognize and bind targeted viral DNA [34]. Next, a trans-activating RNA (tracrRNA) binds the crRNA to process the mature guide RNA (gRNA) and pair up with the endonuclease Cas9 and RNase III to form the Cas9 complex. Once the gRNA binds the complementary target site, it guides the Cas9 nuclease to generate a DSB three nucleotides upstream of the PAM site on the target nucleic acid [35]. CRISPRCas9 thus has the potential to induce precise, site-specific genome editing through the delivery of synthetic single guide (sg) RNAs designed to guide Cas9-mediated cleavage at targeted sites [32].

With the advances in CRISPR-Cas, substantial, ongoing work is using this technique to improve crops through metabolic engineering and regulation of host genes. Several new CRISPR systems, such as Cas12 [36], Cas13a [37, 38], Cas13b [39], and fCas9 [40], are currently in the pipeline. These advances have led to the development of new genome-editing applications that include deactivated Cas9 (dCas9) [41, 42], RNAprocessing Cas9 (RCas9) [37, 43], and Cas9 fusion proteins such as Cas9-cytidine deaminase fusion [44], offering scientists a wide range of CRISPR-based applications.

\section{Approaches to design disease-resistant plants with CRISPR technologies}

Recent advances in CRISPR technology have enabled scientists to develop a broad range of CRISPR variants with different applications, including the modifications described above. In this section, we focus on the CRISPR applications that have been successfully used to engineer disease-resistant plants.

\section{Gene disruption via indels in coding sequences}

This is the most common application of the CRISPR-Cas9 system. It takes advantage of the error-prone behavior of the cellular NHEJ DNA-repair machinery. The result is an insertion or deletion (indel) of one or more nucleotides at the sgRNA-guided site, which introduces a frameshift mutation and disrupts gene production (Fig. 1). This technology has been used in several crops, including essential cereals such as rice and wheat, to introduce traits of interest [28]. In the context of disease resistance, this technology has been used to engineer resistance by disrupting a plant susceptibility $(S)$ gene, which alters the plant-pathogen interaction, leading to reduced pathogen fitness 




on the host plant [45]. A remarkable example of this application was the use of CRIS PR-Cas9 to introduce indels affecting eukaryotic translation initiation factor 4E proteins (eIF4Es), which successfully induced resistance against multiple RNA viruses in Arabidopsis and cucumber (Cucumis sativus) [46, 47].

\section{Gene disruption via indels in promoter regions}

A similar approach can be used to introduce indels in the promoter region instead of the coding region of a plant gene. CRISPR-mediated promoter editing can be applied in two ways: to disrupt the promoter sequence, with the aim of blocking gene 
expression entirely, or to disrupt an effector-binding site, with the aim of disrupting plant susceptibility by preventing a pathogen effector binding to the promoter. The latter approach has been used to modify the promoter of the rice sugar transporter gene OsSWEET14, breaking the connection with the effector from a bacterial blight pathogen and thus leading to blight resistance (Fig. 1) [48-51]. In addition to promoter editing, CRISPR has been demonstrated to alter the gene regulation by targeting upstream open reading frame (ORF) regions and editing cis-regulatory elements [52].

\section{Gene deletion via multiplex sgRNAs}

In CRISPR systems, multiple sgRNAs can be used to introduce multiple DSBs at precise locations in the target genome. For instance, two sgRNAs binding before the start codon and after the stop codon of the gene of interest will produce DSBs at the respective locations. These DSBs then result in the removal of the DNA fragment containing the gene of interest, before the cellular repair NHEJ machinery repairs the DSBs. Because sgRNAs can be designed at any genomic region containing an appropriate PAM trinucleotide sequence, this approach can be and has been used to delete large chromosomal fragments as well as individual genes [53, 54]. This is further facilitated by the development of a rationally designed Cas9, SpCas9-NG, that can recognize NG PAMs, a relaxed stringency compared with the typical NGG PAMs [55]. In the context of engineered pathogen resistance, gene clusters make this approach particularly useful. In $S$ gene clusters, where multiple $S$ genes reside in adjacent chromosomal locations [56], deleting the chromosomal fragment is likely to generate long-term resistance to the target pathogen (Fig. 1).

\section{Gene insertion via homology-directed repair}

The aforementioned CRISPR techniques can be used to generate disease resistance through alteration of $S$ gene(s). However, all plant proteins, including the products of $S$ genes, are important and mostly multifunctional; disrupting these proteins thus comes with costs to plant health and/or productivity. There are alternate approaches, such as the aforementioned cis-regulatory element and promoter editing, to alter the gene expression instead of gene disruption, but it is often necessary to utilize resistance $(R)$ genes against pathogens in cases where the plant-pathogen interaction is not well studied, and the $S$ genes have not been extensively explored. In such cases, the CRISPR toolkit can be used for $R$ gene insertion.

CRISPR-mediated gene insertion works via an alternative route that operates after Cas9 has produced the sgRNA-dictated DSB; this route utilizes the cellular HDR, rather than NHEJ, machinery. A delivery fragment, containing an $R$ gene surrounded by sequence homologous to the DSB ends, is supplemented with Cas9 and the sgRNAs. This cassette guides the HDR-mediated insertion of the $R$ gene between the two DSB sites (Fig. 1). This strategy has been used to introduce one or more genes at precise genomic locations [57]. However, the efficiency of HDR in plants is very low [58], and although new strategies to improve this are under development, currently, this makes gene insertion in plants challenging to implement [59].

In addition to generating disease resistance, CRISPR-mediated gene insertion can be used to study important $S$ gene functions. To demonstrate this, Wang et al. used HDR 
in rice to incorporate green fluorescent protein (GFP) fused in frame with the glutathione S-transferase loci [60]. This application has the potential to be used in studying $S$ gene functions, such as protein localization and the spatiotemporal regulation of $S$ gene expression. Host proteins playing key roles in pathogenicity can be tagged with GFP directly in the genome in order to study their expression and localization during infection. However, such protein modifications can alter their expression and localization, demonstrating the limitations of this approach.

\section{Biomimicking via promoter, allele, or gene replacement}

The concerns surrounding the introduction of foreign DNA into crop products mean that the use of CRISPR for gene insertion is likely to be subjected to lengthy regulatory processes and potentially consumer rejection. To overcome this challenge, CRISPR offers an alternative approach that works on the principle of biomimicking. It has been known for decades that native species and wild relatives of cultivated crops constitute a rich gene pool, especially for resistance against biotic and abiotic stresses [61]. Several recent studies have identified many $R$ genes in the wild relatives of cultivated species and demonstrated the successful transfer of resistance via the identification of an $R$ gene and its transfer to the cultivated crop species $[62,63]$. CRISPR can be used to replace the faulty or poorperforming $R$ gene in a cultivated crop variety with the functional $R$ gene from a diseaseresistant native variety via multiplexed HDR methodology.

Biomimicking refers here to the introduction of CRISPR-mediated mutations in such a way that the sequence of the target gene is converted to the sequence from a diseaseresistant variety. Thus, instead of replacing the whole gene, the researcher introduces only the specific mutations associated with the disease resistance trait, assuming that the nucleotide differences between the gene of interest in the cultivated and wild varieties are not otherwise significant to plant viability and productivity (Fig. 1). This has been mainly achieved by utilizing a CRISPR system designed for targeted nucleotide modification. A fusion of a nuclease-dead Cas9 or nickase with cytidine deaminase can target point mutagenesis with high precision, and this approach has been successfully used in several species for gene modification [64]. The same approach has been used to introduce the N176K substitution encoded by the eIF4E virus-resistance allele (eIF4E1) of Pisum sativum into the Arabidopsis EIF4E1 gene to generate Arabidopsis plants resistant to Clover yellow vein virus (ClYVV) [65]. Recent studies have established the use of synthetic directed evolution to evolve gene variants resistant to splicing inhibitors and herbicides [66-68]. The same approach may be used under the biotic selective pressure to evolve gene variants conferring resistance to selected pathogens.

\section{CRISPR-mediated disease resistance: what has been achieved so far?}

CRISPR technology and its variants have been used for applications in plant science ranging from the study of gene function and protein localization to the introduction of desired traits such as drought tolerance and increased grain size and number. These interesting applications have been covered in recent review articles [26, 28]; here, we focus on CRISPR-mediated engineering of plant disease resistance. Plant diseases are mainly caused by infection with one or more of the main categories of plant pathogens: 
i.e., viruses, bacteria, and fungi. CRISPR technology has been used to engineer resistance against all these major plant pathogen classes (Table 1).

\section{CRISPR-mediated resistance against plant viruses: targeting virus genomes}

Plant viruses are usually managed through improved agricultural practices and the use of virus-resistant crop varieties. Most studies of CRISPR-mediated pathogen resistance in plants have involved resistance to viruses. These studies have demonstrated two main ways to engineer virus resistance: (1) directly targeting virus genome and (2) targeting plant $S$ genes crucial for the development of the viral disease (Fig. 1) [45].

The earliest work on engineered virus resistance used the first approach of directly targeting the virus genome inside plant cells [103]. In two independent studies, Ali et al. targeted multiple single-stranded DNA (ssDNA) geminiviruses: Beet curly top virus, Merremia mosaic virus, Tomato yellow leaf curl virus, and Cotton leaf curl Kokhran virus with its helper betasatellite cotton leaf curl Multan betasatellite, and successfully demonstrated virus targeting by the sgRNA [72, 73, 76]. Within the viral genome, three sites were targeted, two encoding replication-associated protein and capsid protein, and one an intergenic region. Targeting these sites introduced indels in virus genome consequently leading to the lower virus titer and significantly reduced disease symptoms. Baltes et al. and Ji et al. demonstrated similar results, targeting the geminiviruses Bean yellow dwarf virus and Beet severe curly top virus, respectively [74, 75]. Apart from CRISPR-mediated targeting of the viruses, these studies demonstrated limited success in achieving virus resistance in permanent transgenic systems. A similar approach targeting the endogenous Banana streak virus (eBSV) within the genome of plantain banana (Musa spp.) demonstrated that $75 \%$ of the edited events remained asymptomatic compared to the non-edited plants [80].

Direct targeting of virus genomes via CRISPR is a means to engineer resistance against RNA viruses as well. A similar approach targets the viral RNA, instead of DNA, and uses the Cas9 RNA-binding variants, instead of DNA-binding Cas9. Zhang et al. developed $\mathrm{Ni}$ cotiana benthamiana and Arabidopsis plants expressing a FnCas9 targeting Cucumber mosaic virus and Tobacco mosaic virus (TMV) and observed a significant reduction in virus accumulation and reduced symptom development (Fig. 1) [71]. They later utilized a similar strategy to produce resistance to Southern rice black-streaked dwarf virus, an RNA virus targeting rice [70]. In two independent studies, Aman et al. also used an RNAtargeting LwaCas13a (previously known as C2c2) to interfere with the RNA genome of Turnip mosaic virus (TuMV) and demonstrated successful virus RNA targeting in both N. benthamiana [37] and Arabidopsis [69]. Although LwaCas13a showed moderate targeting efficiency against TuMV in Aman et al. studies, these studies showed the great potential of using Cas13 systems in engineering plant immunity against RNA viruses. This led Mahas et al. to further expand the Cas13-mediated virus immunity by screening various Cas13 variants and identifying Cas13d system (CasRx) as the most robust Cas13 ortholog to target and interfere with different RNA viruses, including TMV, Potato virus X (PVX), and TuMV (Fig. 1) [104]. The impressive efficiency of Cas13 for targeting and interfering with RNA viruses in plants could be exploited to potentially provide immunity against the economically significant plant-infecting viroids. Whether Cas13 could successfully target and degrade circular, highly compact and secondary structure-rich RNA viroid genomes remain to be seen [105]. 
Table 1 A summary of the studies on CRISPR-mediated plant disease resistance

\begin{tabular}{|c|c|c|c|c|c|c|}
\hline $\begin{array}{l}\text { Pathogen } \\
\text { type }\end{array}$ & Plant(s) & $\begin{array}{l}\text { Desired } \\
\text { modification }\end{array}$ & $\begin{array}{l}\text { Targeted DNA/ } \\
\text { RNA }\end{array}$ & $\begin{array}{l}\text { Targeted } \\
\text { pathogen(s)/ } \\
\text { disease(s) }\end{array}$ & Results & Reference \\
\hline \multirow[t]{16}{*}{ m } & Arabidopsis & $\begin{array}{l}\text { Virus RNA } \\
\text { genome } \\
\text { disruption }\end{array}$ & Virus RNA genome & Turnip mosaic virus & $\begin{array}{l}\text { Indels in virus } \\
\text { RNA }\end{array}$ & [69] \\
\hline & $\begin{array}{l}N . \\
\text { benthamiana }\end{array}$ & $\begin{array}{l}\text { Virus RNA } \\
\text { genome } \\
\text { disruption }\end{array}$ & Virus RNA genome & Turnip mosaic virus & $\begin{array}{l}\text { Indels in virus } \\
\text { RNA }\end{array}$ & [37] \\
\hline & $\begin{array}{l}\text { Rice, } N \text {. } \\
\text { benthamiana }\end{array}$ & $\begin{array}{l}\text { Virus RNA } \\
\text { genome } \\
\text { disruption }\end{array}$ & Virus RNA genome & $\begin{array}{l}\text { Southern rice black- } \\
\text { streaked dwarf virus, } \\
\text { Tobacco mosaic virus }\end{array}$ & $\begin{array}{l}\text { Reduction in } \\
\text { virus levels } \\
\text { and disease } \\
\text { symptoms }\end{array}$ & [70] \\
\hline & $\begin{array}{l}\text { Arabidopsis, } \\
N . \\
\text { benthamiana }\end{array}$ & $\begin{array}{l}\text { Virus RNA } \\
\text { genome } \\
\text { disruption }\end{array}$ & Virus RNA genome & $\begin{array}{l}\text { Cucumber mosaic virus, } \\
\text { Tobacco mosaic virus }\end{array}$ & $\begin{array}{l}\text { Reduction in } \\
\text { virus levels } \\
\text { and disease } \\
\text { symptoms }\end{array}$ & [71] \\
\hline & $\begin{array}{l}N . \\
\text { benthamiana }\end{array}$ & $\begin{array}{l}\text { Virus DNA } \\
\text { disruption }\end{array}$ & $\begin{array}{l}\text { Virus DNA } \\
\text { Rep, IR, and Cp }\end{array}$ & $\begin{array}{l}\text { Beet curly top virus, } \\
\text { Merremia mosaic virus, } \\
\text { Tomato yellow leaf curl } \\
\text { virus }\end{array}$ & $\begin{array}{l}\text { Indels in virus } \\
\text { DNA }\end{array}$ & {$[72]$} \\
\hline & $\begin{array}{l}N . \\
\text { benthamiana }\end{array}$ & $\begin{array}{l}\text { Virus DNA } \\
\text { disruption }\end{array}$ & $\begin{array}{l}\text { Virus DNA and } \\
\text { satellite sequences }\end{array}$ & $\begin{array}{l}\text { Cotton leaf curl Kokhran } \\
\text { virus, Tomato yellow } \\
\text { leaf curl Sardinian virus, } \\
\text { Tomato yellow leaf curl } \\
\text { virus, Merremia mosaic } \\
\text { virus, BCTV-Logan, } \\
\text { BCTV-Worland }\end{array}$ & $\begin{array}{l}\text { Indels in virus } \\
\text { DNA }\end{array}$ & [73] \\
\hline & $\begin{array}{l}N . \\
\text { benthamiana }\end{array}$ & $\begin{array}{l}\text { Virus DNA } \\
\text { disruption }\end{array}$ & $\begin{array}{l}\text { Virus DNA } \\
\text { Rep A/Rep and LIR }\end{array}$ & Bean yellow dwarf virus & $\begin{array}{l}\text { Indels in virus } \\
\text { DNA, } \\
\text { resistance to } \\
\text { virus }\end{array}$ & [74] \\
\hline & $\begin{array}{l}\text { Arabidopsis, } \\
N . \\
\text { benthamiana }\end{array}$ & $\begin{array}{l}\text { Virus DNA } \\
\text { disruption }\end{array}$ & $\begin{array}{l}\text { Virus DNA } \\
\text { Rep, IR, and CP }\end{array}$ & $\begin{array}{l}\text { Beet severe curly top } \\
\text { virus }\end{array}$ & $\begin{array}{l}\text { Indels in virus } \\
\text { DNA, } \\
\text { resistance to } \\
\text { virus }\end{array}$ & {$[75]$} \\
\hline & $\begin{array}{l}\text { Tomato, } N \text {. } \\
\text { benthamiana }\end{array}$ & $\begin{array}{l}\text { Virus DNA } \\
\text { disruption }\end{array}$ & $\begin{array}{l}\text { Virus DNA } \\
\text { Rep, IR, and Cp }\end{array}$ & $\begin{array}{l}\text { Tomato yellow leaf curl } \\
\text { virus }\end{array}$ & $\begin{array}{l}\text { Indels in virus } \\
\text { DNA, } \\
\text { resistance to } \\
\text { virus }\end{array}$ & [76] \\
\hline & $\begin{array}{l}N . \\
\text { benthamiana }\end{array}$ & $\begin{array}{l}\text { Virus DNA } \\
\text { disruption }\end{array}$ & $\begin{array}{l}\text { Multiplex editing } \\
\text { at Rep and IR }\end{array}$ & $\begin{array}{l}\text { Cotton leaf curl Multan } \\
\text { virus }\end{array}$ & $\begin{array}{l}\text { Significantly } \\
\text { low virus } \\
\text { accumulation } \\
\text { and decreased } \\
\text { disease } \\
\text { symptoms }\end{array}$ & [77] \\
\hline & Cassava & $\begin{array}{l}\text { Virus DNA } \\
\text { disruption }\end{array}$ & $A C 2$ and $A C 3$ & $\begin{array}{l}\text { African cassava mosaic } \\
\text { virus }\end{array}$ & $\begin{array}{l}\text { Indels in virus } \\
\text { DNA but no } \\
\text { virus } \\
\text { resistance }\end{array}$ & [78] \\
\hline & $\begin{array}{l}N . \\
\text { benthamiana }\end{array}$ & $\begin{array}{l}\text { Virus DNA } \\
\text { disruption }\end{array}$ & $\begin{array}{l}\text { Multiplex editing } \\
\text { at virus DNA Rep, } \\
\mathbb{I} \text {, and } C p\end{array}$ & Chilli leaf curl virus & $\begin{array}{l}\text { Significantly } \\
\text { low virus } \\
\text { accumulation } \\
\text { and decreased } \\
\text { disease } \\
\text { symptoms }\end{array}$ & [79] \\
\hline & Banana & $\begin{array}{l}\text { Virus DNA } \\
\text { disruption }\end{array}$ & $\begin{array}{l}\text { Virus sequences in } \\
\text { the host plantain } \\
\text { genome }\end{array}$ & $\begin{array}{l}\text { Endogenous banana } \\
\text { streak virus }\end{array}$ & $\begin{array}{l}75 \% \text { of } \\
\text { pl0ants remain } \\
\text { asymptomatic }\end{array}$ & {$[80]$} \\
\hline & & Biomimicking $^{\text {a }}$ & Eiftel & Clover yellow vein virus & $\begin{array}{l}\text { Reduced virus } \\
\text { accumulation }\end{array}$ & {$[65]$} \\
\hline & Rice & Biomimicking $^{\text {a }}$ & Eif4g & $\begin{array}{l}\text { Rice tungro spherical } \\
\text { virus }\end{array}$ & $\begin{array}{l}\text { Resistance to } \\
\text { virus }\end{array}$ & [81] \\
\hline & Cassava & $\begin{array}{l}\text { Gene } \\
\text { disruption }\end{array}$ & $n C B P-1, n C B P-2$ & $\begin{array}{l}\text { Cassava brown streak } \\
\text { disease }\end{array}$ & $\begin{array}{l}\text { Suppressed } \\
\text { disease }\end{array}$ & [82] \\
\hline
\end{tabular}


Table 1 A summary of the studies on CRISPR-mediated plant disease resistance (Continued)

\begin{tabular}{|c|c|c|c|c|c|c|}
\hline $\begin{array}{l}\text { Pathogen } \\
\text { type }\end{array}$ & Plant(s) & $\begin{array}{l}\text { Desired } \\
\text { modification }\end{array}$ & $\begin{array}{l}\text { Targeted DNA/ } \\
\text { RNA }\end{array}$ & $\begin{array}{l}\text { Targeted } \\
\text { pathogen(s)/ } \\
\text { disease(s) }\end{array}$ & Results & Reference \\
\hline & & & & & symptoms & \\
\hline & Arabidopsis & $\begin{array}{l}\text { Gene } \\
\text { disruption }\end{array}$ & EIF4E & Turnip mosaic virus & $\begin{array}{l}\text { Resistance to } \\
\text { virus }\end{array}$ & [47] \\
\hline & Cucumber & $\begin{array}{l}\text { Gene } \\
\text { disruption }\end{array}$ & elF4E & $\begin{array}{l}\text { Cucumber vein } \\
\text { yellowing virus } \\
\text { (ipomovirus), Zucchini } \\
\text { yellow mosaic virus, and } \\
\text { Papaya ring spot } \\
\text { mosaic virus-W } \\
\text { (potyviruses) }\end{array}$ & $\begin{array}{l}\text { Resistance to } \\
\text { three viruses }\end{array}$ & {$[46]$} \\
\hline \multirow[t]{12}{*}{ Fungus } & Tomato & $\begin{array}{l}\text { Gene } \\
\text { disruption }\end{array}$ & $\begin{array}{l}\text { Multiplex gRNA at } \\
\text { Pmr4 }\end{array}$ & $\begin{array}{l}\text { Powdery mildew } \\
\text { caused by Oidium } \\
\text { neolycopersici }\end{array}$ & $\begin{array}{l}\text { Significant } \\
\text { reduction in } \\
\text { mildew } \\
\text { symptoms }\end{array}$ & [83] \\
\hline & Tomato & $\begin{array}{l}\text { Gene } \\
\text { disruption }\end{array}$ & SIMapk3 & Botrytis cinerea & $\begin{array}{l}\text { Increased } \\
\text { resistance to } \\
\text { B. cinerea }\end{array}$ & [84] \\
\hline & Tomato & $\begin{array}{l}\text { Gene } \\
\text { disruption }\end{array}$ & Solyc08g075770 & Fusarium wilt & $\begin{array}{l}\text { Tolerance to } \\
\text { fusarium wilt }\end{array}$ & [85] \\
\hline & Rice & $\begin{array}{l}\text { Gene } \\
\text { disruption }\end{array}$ & $\begin{array}{l}\text { Single and } \\
\text { multiplex gRNA at } \\
\text { OsERF922 }\end{array}$ & $\begin{array}{l}\text { Rice blast caused by } \\
\text { Magnaporthe oryzae }\end{array}$ & $\begin{array}{l}\text { Significantly } \\
\text { decreased } \\
\text { blast lesions }\end{array}$ & [86] \\
\hline & Grape & $\begin{array}{l}\text { Gene } \\
\text { disruption }\end{array}$ & VVWRKY52 & B. cinerea & $\begin{array}{l}\text { Increased } \\
\text { resistance to } \\
\text { B. cinerea }\end{array}$ & [87] \\
\hline & Tomato & $\begin{array}{l}\text { Gene } \\
\text { disruption }\end{array}$ & SIMlo1 & Powdery mildew & $\begin{array}{l}\text { Resistance to } \\
\text { powdery } \\
\text { mildew }\end{array}$ & [88] \\
\hline & Banana & $\begin{array}{l}\text { Gene } \\
\text { insertion }\end{array}$ & RGA2, Ced 9 & $\begin{array}{l}\text { Fusarium wilt caused } \\
\text { by Fusarium oxysporum } \\
\text { f. sp. cubense tropical } \\
\text { race } 4 \text { (TR4) }\end{array}$ & $\begin{array}{l}\text { Significant } \\
\text { reduction in } \\
\text { disease }\end{array}$ & [89] \\
\hline & Rice & $\begin{array}{l}\text { Gene } \\
\text { disruption }\end{array}$ & OSMPK5 & $\begin{array}{l}\text { Fungal (Magnaporthe } \\
\text { grisea) and bacterial } \\
\text { (Burkholderia glumae) } \\
\text { pathogens }\end{array}$ & $\begin{array}{l}\text { Indels in the } \\
\text { target; } \\
\text { resistance not } \\
\text { confirmed }\end{array}$ & [90] \\
\hline & Grape & $\begin{array}{l}\text { Gene } \\
\text { disruption }\end{array}$ & Mlo-7 & Powdery mildew & $\begin{array}{l}\text { Indels in the } \\
\text { target; } \\
\text { resistance not } \\
\text { confirmed }\end{array}$ & [91] \\
\hline & Wheat & $\begin{array}{l}\text { Gene } \\
\text { disruption }\end{array}$ & $\begin{array}{l}\text { TaMlo-A1, TaMlo- } \\
B 1 \text {, and TaMlo-D1 }\end{array}$ & Powdery mildew & $\begin{array}{l}\text { High tolerance } \\
\text { to powdery } \\
\text { mildew }\end{array}$ & [92] \\
\hline & Wheat & $\begin{array}{l}\text { Gene } \\
\text { disruption }\end{array}$ & TaMlo & Powdery mildew & $\begin{array}{l}\text { Indels in the } \\
\text { target; } \\
\text { resistance not } \\
\text { confirmed }\end{array}$ & [30] \\
\hline & Wheat & $\begin{array}{l}\text { Gene } \\
\text { disruption }\end{array}$ & $\begin{array}{l}\text { TaEdr1 (three } \\
\text { homologs) }\end{array}$ & Powdery mildew & $\begin{array}{l}\text { Resistance to } \\
\text { powdery } \\
\text { mildew }\end{array}$ & [93] \\
\hline \multirow[t]{3}{*}{ Bacteria } & Rice & $\begin{array}{l}\text { Gene } \\
\text { disruption }\end{array}$ & OsSWEET13 & $\begin{array}{l}\text { Bacterial blight caused } \\
\text { by Xanthomonas } \\
\text { oryzae pv. Oryzae (Xoo) }\end{array}$ & $\begin{array}{l}\text { Resistance not } \\
\text { confirmed }\end{array}$ & [94] \\
\hline & Rice & $\begin{array}{l}\text { Gene } \\
\text { disruption }\end{array}$ & OsSWEET11 & Bacterial blight & $\begin{array}{l}\text { Enhanced } \\
\text { resistance to } \\
\text { Xoo }\end{array}$ & [95] \\
\hline & Rice & $\begin{array}{l}\text { Gene and } \\
\text { promoter } \\
\text { disruption }\end{array}$ & $\begin{array}{l}\text { TALE-binding } \\
\text { elements (EBES) in } \\
\text { OsSWEET13 } \\
\text { promoter, }\end{array}$ & $\begin{array}{l}\text { Bacterial blight caused } \\
\text { by Xoo }\end{array}$ & $\begin{array}{l}\text { Broad- } \\
\text { spectrum } \\
\text { resistance } \\
\text { against }\end{array}$ & [50] \\
\hline
\end{tabular}


Table 1 A summary of the studies on CRISPR-mediated plant disease resistance (Continued)

\begin{tabular}{|c|c|c|c|c|c|c|}
\hline $\begin{array}{l}\text { Pathogen } \\
\text { type }\end{array}$ & Plant(s) & $\begin{array}{l}\text { Desired } \\
\text { modification }\end{array}$ & $\begin{array}{l}\text { Targeted DNA/ } \\
\text { RNA }\end{array}$ & $\begin{array}{l}\text { Targeted } \\
\text { pathogen(s)/ } \\
\text { disease(s) }\end{array}$ & Results & Reference \\
\hline & & & $\begin{array}{l}\text { OsSWEETT11, and } \\
\text { OsSWEEt14 genes }\end{array}$ & & $\begin{array}{l}\text { multiple Xoo } \\
\text { strains }\end{array}$ & \\
\hline & Rice & $\begin{array}{l}\text { Promoter } \\
\text { disruption }\end{array}$ & $\begin{array}{l}\text { OsSWEET11, } \\
\text { OsSWEET13, and } \\
\text { OsSWEET14 }\end{array}$ & Bacterial blight & $\begin{array}{l}\text { Increased } \\
\text { resistance to } \\
\text { bacterial } \\
\text { blight; } \\
\text { confirmed in } \\
\text { field trials }\end{array}$ & [51] \\
\hline & Apple & $\begin{array}{l}\text { Gene } \\
\text { disruption }\end{array}$ & $\begin{array}{l}\text { DIPM-1, DIPM-2, } \\
\text { and DIPM-4 }\end{array}$ & $\begin{array}{l}\text { Fire blight disease } \\
\text { (caused by Erwinia } \\
\text { amylovora) }\end{array}$ & $\begin{array}{l}\text { Indels in the } \\
\text { target; } \\
\text { resistance not } \\
\text { confirmed }\end{array}$ & [91] \\
\hline & Rice & $\begin{array}{l}\text { Promoter } \\
\text { disruption }\end{array}$ & $\begin{array}{l}\text { OsSWEET11, } \\
\text { OsSWEET14 }\end{array}$ & Bacterial blight & $\begin{array}{l}\text { Indels in } \\
\text { promoter; } \\
\text { disease } \\
\text { resistance not } \\
\text { confirmed }\end{array}$ & {$[96]$} \\
\hline & Tomato & Gene repair & Jaz2 & $\begin{array}{l}\text { Bacterial speck disease } \\
\text { caused by } \\
\text { Pseudomonas syringae } \\
\text { pv. tomato DC } 3000\end{array}$ & $\begin{array}{l}\text { Resistance to } \\
\text { bacterial speck } \\
\text { disease }\end{array}$ & [97] \\
\hline & Tomato & $\begin{array}{l}\text { Gene } \\
\text { disruption }\end{array}$ & Dmr6 & $\begin{array}{l}\text { Pseudomonas syringae, } \\
\text { Phytophthora capsici, } \\
\text { and Xanthomonas spp. }\end{array}$ & $\begin{array}{l}\text { Resistance to } \\
\text { P. syringae, P. } \\
\text { capsici, and } \\
\text { Xanthomonas } \\
\text { spp. }\end{array}$ & [98] \\
\hline & Grapefruit & $\begin{array}{l}\text { Promoter } \\
\text { disruption }\end{array}$ & CsLOB1 & Citrus canker & $\begin{array}{l}\text { Significantly } \\
\text { reduced } \\
\text { canker } \\
\text { symptoms }\end{array}$ & [99] \\
\hline & $\begin{array}{l}\text { Wanjincheng } \\
\text { orange }\end{array}$ & $\begin{array}{l}\text { Promoter } \\
\text { disruption }\end{array}$ & CsLOB1 & Citrus canker & $\begin{array}{l}\text { Disease } \\
\text { severity } \\
\text { decreased by } \\
83.2-98.3 \%\end{array}$ & [100] \\
\hline \multirow[t]{2}{*}{ Oomycete } & Papaya & $\begin{array}{l}\text { Gene } \\
\text { disruption }\end{array}$ & PpalEPIC8 & Phytophthora palmivora & $\begin{array}{l}\text { Increased } \\
\text { resistance } \\
\text { against } P \text {. } \\
\text { palmivora }\end{array}$ & [101] \\
\hline & $\begin{array}{l}\text { Theobroma } \\
\text { cacao }\end{array}$ & $\begin{array}{l}\text { Gene } \\
\text { disruption }\end{array}$ & TCNPR3 & Phytophthora tropicalis & $\begin{array}{l}\text { Increased } \\
\text { resistance } \\
\text { against } P \text {. } \\
\text { tropicalis }\end{array}$ & [102] \\
\hline
\end{tabular}

aiomimicking refers here to the introduction of CRISPR-mediated mutations in such a way that the sequence of a target gene in disease-susceptible variety is converted to the sequence from a disease-resistant variety. Thus, instead of replacing the whole gene, the researcher introduces only the specific mutations associated with the disease resistance trait, assuming that the nucleotide differences between the gene of interest in the cultivated and wild varieties are not otherwise significant to plant viability and productivity

Ali et al. highlighted a potential problem with the system by demonstrating the possibility of virus escape from the CRISPR-edited plants [73]. Virus escape was later confirmed by Mehta et al., whose attempt to engineer resistance against African cassava mosaic virus in permanent transgenic cassava (Manihot esculenta) lines failed to establish resistance [78]. Further investigation revealed that $33-48 \%$ of edited virus genomes carried a conserved single-nucleotide mutation that conferred resistance to CRISPRCas9 cleavage and generated virus escape mutants (Fig. 1) [78]. A proposed strategy to counter this limitation is the use of a multiplex CRISPR system to introduce multiple DSBs in virus DNA, which should be less prone to NHEJ-repaired functional virus mutations and, in turn, to the development of escape mutants [106]. A recent study has 
indeed demonstrated the targeting of a geminivirus, Chilli leaf curl virus, at multiple sgRNA target sites and a significant reduction in viral DNA accumulation [79]. Subsequent transient assays confirmed a significant decrease in viral DNA accumulation and disease symptoms [79]. Similar results were obtained by Yin et al., who used a multiplex CRISPR system to target Cotton leaf curl Multan virus at multiple genomic sites (Rep and IR) and achieved successful resistance against virus in transgenic $N$. benthamiana plants [77]. Further studies in crop plants and tests in field trials will be needed to validate these findings. Although alternative strategies such as multiplex editing are aimed at addressing this, the potential for viral escape and the evolution of CRISPR-resistant viruses remain the biggest concerns with this approach and limit the applications of direct viral-genome-targeting CRISPR technology [106, 107].

\section{CRISPR-mediated resistance against plant viruses: targeting plant $S$ gene(s)}

CRISPR-mediated targeting of $S$ gene/s avoids the limitations associated with direct viral genome targeting, as with this approach, the sgRNA targets the plant gene instead of the viral genome, which is more prone to evasion because of its high copy number and high recombination rate. Moreover, $S$ gene disruption can be performed following transgene-free CRISPR protocols. Thus, when $S$ genes are known and well characterized, CRISPR-mediated targeting of $S$ genes appears to be a better approach to engineer viral immunity.

The $S$ genes most widely targeted in CRISPR-mediated engineering of virus resistance are the eIF4E genes, which encode cap-binding proteins essential for the cellular infection cycle of various RNA potyviruses. The potyviral 5 '-terminal protein VPg interacts with eIF4Es, and blocking this interaction triggers immunity against potyviruses in various plant species. In two independent studies, CRISPR-mediated eIF4E targeting yielded successful resistance against multiple viruses, including TuMV, Cucumber vein yellowing virus, Zucchini yellow mosaic virus, and Papaya ring spot mosaic virus-W, in Arabidopsis and cucumber [46, 47]. Later studies targeting eIF4E isoforms in other important crop species produced broad-spectrum virus resistance in a variety of hosts. Gomez et al. targeted the eIF4E isoforms in cassava, novel cap-binding protein-1 (nCBP-1) and nCBP-2, and showed that this significantly suppressed the symptoms of Cassava brown streak virus disease [82]. Furthermore, a biomimicking approach generated CRISPR-mediated mutations in Arabidopsis eIF4E1 that converted its sequence to match the $P$. sativum eIF4E virus-resistance allele, successfully introducing resistance against ClYVV [65]. Likewise, Macovei et al. demonstrated resistance in rice against rice Tungro spherical virus through biomimicking of eIF4G alleles [81]. In summary, multiple CRISPR systems have utilized $S$ genes to achieve virus resistance in a number of crop species.

\section{CRISPR-mediated resistance against bacteria}

The most successful example of CRISPR-mediated introduction of bacterial resistance in crops is by using OsSWEET gene/s to trigger immunity against bacterial blight caused by Xanthomonas oryzae pv. oryzae. The rice SWEET clade III family contains the genes OsSWEET11, OsSWEET13, and OSSWEET14, which encode sugar transporters mediating glucose and sucrose export; SWEET gene induction by transcription activator-like effectors (TALEs, the bacterial proteins used to develop TALENS) 
triggers sugar release into the apoplast, providing a nutrient source for the pathogen, and thus, these genes act as $S$ genes. Genome editing via TALENs had already demonstrated that rice bearing one or more OSSWEET knock-outs shows resistance to bacterial blight $[48,49]$. Further studies identified the TALE-binding elements (EBEs) in the OsSWEET promoters as key virulence factors in recessive OsSWEET-mediated resistance [50, 94]. Recently, a comprehensive study introduced mutations into the promoters of all three OSSWEET genes via CRISPR-Cas9 and reported that this resulted in broad-spectrum resistance to bacterial blight (Fig. 1) [51].

In tomato (Solanum lycopersicum), bacterial speck disease is caused by Pseudomonas syringae pv. tomato DC 3000, which produces coronatine that, with the co-receptor JAZ2, stimulates stomata opening and facilitates leaf colonization by the bacteria. CRIS PR-Cas9 has been used to edit SlJAZ2 to produce a dominant allele encoding a variant of JAZ2 that lacks the C-terminal Jas domain, which prevents stomatal reopening and provides resistance to bacterial speck disease [97]. Another Arabidopsis gene, DMR6 (DOWNY MILDEW RESISTANT 6), is strongly associated with salicylic acid regulation and, in turn, with pathogen infection in plants. CRISPR has been used to target its tomato ortholog, SlDmr6-1, conferring broad-spectrum resistance against multiple pathogens including P. syringae, Phytophthora capsici, and Xanthomonas spp. [98].

One of the most economically important bacterial diseases for which CRISPR provides a resistance solution is citrus canker. This infection, caused by Xanthomonas citri subsp. citri, is considered among the most destructive diseases of citrus, causing yield losses in citrus-growing regions worldwide. The main TALE of the bacterium, PthA4, specifically binds to the effector-binding element in the promoter of a citrus canker-susceptibility gene, LATERAL ORGAN BOUNDARIES 1 (CsLOB1), and activates its expression to favor citrus canker development [99]. Two independent studies have demonstrated that CRISPR-mediated editing of CsLOB1 leads to significantly reduced citrus canker symptoms in two citrus species, grapefruit (Citrus $\times$ paradisi) [100] and Wanjincheng orange (Citrus sinensis Osbeck) [99].

\section{CRISPR-mediated resistance against fungi}

Fungal pathogens are causal agents of around 30\% of emerging plant diseases [108] and infect numerous economically important food crops (Table 1). CRISPR systems have been used to target and disrupt the plant $S$ genes for fungal pathogen susceptibility. Barley Mildew resistance locus $O(M l o)$ encodes a membrane-associated protein that is essential for fungal pathogen penetration of the host epidermal cells [109], and multiple studies indicated that mutation of Mlo triggers plant immunity against powdery mildew [110-112]. Mutating $M l o$ via CRISPR-Cas9 also conferred powdery mildew resistance in wheat [30, 88, 92] and tomato non-transgenic systems [88]. Another powdery mildew $S$ gene, Edr1 (Enhanced disease resistance 1), which encodes a Raf-like mitogen-activated protein, has also been targeted via CRISPR-Cas9, resulting in a significant reduction in powdery mildew in wheat [93]. Recently, CRISPR-mediated targeting of the Powdery mildew resistance 4 (Pmr4) S gene, whose resistance mechanism is not completely understood, has been demonstrated to cause a significant reduction of powdery mildew disease symptoms in tomato [83].

CRISPR has been used for gene disruption in rice targeting OsERF922, which encodes

a key ethylene-responsive factor that is involved in the modulation of biotic stress 
response and is a negative regulator of blast resistance in rice. Single or multiple sgRNAs generated OsERF922 mutant rice lines that showed tolerance against rice blast, with significantly decreased blast lesions compared to the controls [86]. Prihatna et al. used CRISPR to study the reduced mycorrhizal colonization $(r m c)$ tomato mutant lines, which contain a chromosomal deletion affecting five genes that makes the plants susceptible to fusarium wilt. CRISPR-Cas9-mediated knock-out and complementation of one of the five genes, Solyc08g075770, indicated that it is involved in this fusarium wilt tolerance [85]. Wang et al. used CRISPR to target and disrupt a transcription factor gene, VvWRKY52, with a critical role in plant response to biotic stress in the grape (Vitis vinifera) cultivar Thompson Seedless and observed increased resistance against the fungal pathogen Botrytis cinerea [87]. Likewise, Zhang et al. demonstrated that CRISPR-mediated disruption of the gene encoding tomato mitogen-activated protein kinase 3 (SIMAPK3), which regulates the accumulation of reactive oxygen species (ROS), generated tomato plants resistant to B. cinerea [84].

\section{CRISPR-mediated resistance against oomycete}

Apart from the major viral, bacterial, and fungal pathogens, CRISPR has also been used to address other biotic stresses, such as oomycete infection (Fig. 1). Phytophthora palmivora is a destructive oomycete pathogen of papaya; the CRISPR-Cas9 system was used to develop papaya plant mutant for a functional cysteine protease inhibitor (PpaIEPIC8), which led to increased resistance against $P$. palmivora [101]. Likewise, resistance has been engineered against another oomycete pathogen, Phytophthora tropicalis, in Theobroma cacao, the tropical tree that produces cocoa beans [102].

\section{Limitations and future prospects}

CRISPR is being increasingly used to introduce desired traits, including disease resistance, in numerous economically important crop species. Several independent studies have demonstrated successful CRISPR-mediated engineered resistance and, in some cases, broad-spectrum resistance against multiple pathogens (Table 1) [50, 51]. Moreover, these demonstrations of CRISPR-mediated disease resistance have not been limited only to the laboratory or greenhouse: several CRISPR crop varieties are in the pipeline for commercialization and at least one product, false flax (C. sativa) with enhanced omega-3 oil, is reaching the market in record time in the USA [25]. This is an indication that CRISPR crops and their products will reach consumers in the near future, demonstrating that the exciting applications we have discussed here have great potential in the development of future commercial crop varieties.

There have been several recent developments in the CRISPR technology that can be directly implemented in disease-resistant crop production: for example, generating gene-edited dicotyledonous plants through de novo meristem induction and eliminating time-consuming tissue culture steps [113], using temperature-tolerant CRISPR/ LbCas12a to increase the targeting and efficiency [114], enabling large DNA insertions (up to $2 \mathrm{~kb}$ ) with precision in rice [115], and applying heat-inducible CRISPR system to increase the efficiency of gene targeting in maize [116]. Chromosome engineering in crops is another exciting recent development enabling controlled restructuring of plant genomes [117] and breaking genetic linkage via somatic chromosome engineering 
[118]. Taken together, these developments would further streamline the transfer of resistance genes to elite cultivars.

Notably, the most economically important plant virus diseases are caused by geminiviruses, and all studies to date of CRISPR-mediated geminivirus resistance have used direct virus DNA targeting. This approach has its limitations, however, owing to the possibility of virus escape and generation of resistance-blocking strains (see the "CRIS PR-mediated resistance against plant viruses: targeting virus genomes" section). The most probable solution is the utilization of host susceptibility factors involved specifically in the plant-geminivirus interaction. However, whereas there are well-characterized $S$ genes for other pathogens, such as Mlo for powdery mildew and eIF4E for potyviruses, such high-performance, precise $S$ genes are not currently available for geminiviruses. Considerable work has been done to understand the process of geminivirus infection, and several relevant plant genes have been identified; these comprehensive review articles summarize host susceptibility factors that may be potential target $S$ genes for engineering geminivirus resistance, an important strategy to be pursued in the future [119-122].

Given that the CRISPR technology was developed very recently, and it takes several years to develop commercial crop varieties and move them through the standard regulatory procedures, there is a need to revise the regulatory timeline. Moreover, the current legal framework within the EU regulates CRISPR crops as GM crops, even when the products are transgene-free and contain no foreign DNA [123, 124]. These regulatory frameworks increase the time and cost of variety development. Scientists and policymakers need to work together and devise comprehensive plans for CRISPR crops integration. Excellent examples of such efforts include a science-based regulatory framework designed for engineered crops [125] and a GMO opt-in mechanism laid out for the EU [126].

Apart from improvements to the regulatory and policy environment, several technological improvements are also needed to facilitate the development and testing of CRIS PR crops. A technological bottleneck to CRISPR in plants is the low innate HDR efficiency, which hinders several intended applications, such as gene replacement and large chromosomal deletions. Although new strategies are being developed to improve HDR efficiency in plants $[57,58,127]$, this hurdle currently makes gene-insertion applications in plants challenging. Developing novel methods for the delivery of the genome engineering machinery into germline cells will unlock the potential of this technology to generate foreign DNA-free edited genomes. It will enable engineering wild relatives or germplasm currently used in agriculture but recalcitrant for transformation. Finally, extensive field trials are needed to test the performance of these crops, at least for sustained resistance, and also for other productivity traits that may be compromised if, for example, a multifunctional $S$ gene is disrupted.

\section{Supplementary Information}

The online version contains supplementary material available at https://doi.org/10.1186/s13059-020-02204-y.

Additional file 1. Review history. 


\section{Peer review information}

Kevin Pang was the primary editor of this article and managed its editorial process and peer review in collaboration with the rest of the editorial team.

\section{Review history}

The review history is available as Additional file 1.

\section{Authors' contributions}

SSZ drafted the first version of the manuscript. AM drafted the figure and wrote the corresponding sections. MM and HV critically read and revised the manuscript. All authors read and approved the final manuscript.

\section{Funding}

This publication is based upon work supported by the King Abdullah University of Science and Technology (KAUST) Office of Sponsored Research (OSR) under Award No. OSR-2015-CRG4-2647. The authors acknowledge financial support from the Belgian FNRS grant M.i.S. F.4515.17 to H.V. and grant 1.B456.20 to S.S.Z. and H.V.

\section{Ethics approval and consent to participate}

Not applicable.

\section{Competing interests}

The authors declare that they have no competing interests.

\section{Author details}

1Plant Genetics, TERRA Teaching and Research Center, Gembloux Agro-Bio Tech, University of Liège, Gembloux Belgium. 'Laboratory for Genome Engineering and Synthetic Biology, Division of Biological Sciences, 4700 King Abdullah University of Science and Technology, Thuwal 23955-6900, Saudi Arabia. ${ }^{3}$ Laboratory of Tropical Crop Improvement, Division of Crop Biotechnics, Biosystems Department, KU Leuven, Leuven, Belgium.

Received: 25 August 2020 Accepted: 13 November 2020

Published online: 30 November 2020

\section{References}

1. Hickey LT, et al. Breeding crops to feed 10 billion. Nat Biotechnol. 2019;37(7):744-54.

2. Ray DK, et al. Recent patterns of crop yield growth and stagnation. Nat Commun. 2012;3:1293.

3. Ritchie, H. and M. Roser, Land Use. 2019. Published online at OurWorldlnData.org. Retrieved from: 'https:// ourworldindata.org/land-use'. Accessed 22 Nov 2020.

4. Añel JA, et al. Understanding climate change from a global analysis of city analogues. Plos One. 2019;14(7):e0217592.

5. Newbery F, Qi A, Fitt BD. Modelling impacts of climate change on arable crop diseases: progress, challenges and applications. Curr Opin Plant Biol. 2016:32:101-9.

6. Roussi A. Why gigantic locust swarms are challenging governments and researchers. Nature. 2020;579(7799):330.

7. Pingali PL. Green revolution: impacts, limits, and the path ahead. Proc Natl Acad Sci U S A. 2012;109(31):12302-8.

8. Tester M, Langridge P. Breeding technologies to increase crop production in a changing world. Science. 2010;327(5967): 818-22.

9. Bailey-Serres J, et al. Genetic strategies for improving crop yields. Nature. 2019;575(7781):109-18.

10. Ezezika OC, et al. Factors influencing agbiotech adoption and development in sub-Saharan Africa. Nat Biotechnol. 2012; 30(1):38-40.

11. Zaidi SS, et al. New plant breeding technologies for food security. Science. 2019;363(6434):1390-1.

12. Ghosh $\mathrm{S}$, et al. Speed breeding in growth chambers and glasshouses for crop breeding and model plant research. Nat Protoc. 2018;13(12):2944-63.

13. Watson A, et al. Speed breeding is a powerful tool to accelerate crop research and breeding. Nat Plants. 2018;4(1):23-9.

14. Nguyen KL, et al. Next-generation sequencing accelerates crop gene discovery. Trends Plant Sci. 2019;24(3):263-74.

15. Barabaschi D, et al. Next generation breeding. Plant Sci. 2016;242:3-13.

16. Zorrilla-Fontanesi, Y., et al., Strategies to revise agrosystems and breeding for Fusarium 1 wilt control of banana. Nature Foods, 2020. In Press.

17. He Y, Zhao Y. Technological breakthroughs in generating transgene-free and genetically stable CRISPR-edited plants. aBIOTECH. 2019;1(1):88-96.

18. Liang Z, et al. Genome editing of bread wheat using biolistic delivery of CRISPR/Cas9 in vitro transcripts or ribonucleoproteins. Nat Protoc. 2018;13:413-30.

19. Woo JW, et al. DNA-free genome editing in plants with preassembled CRISPR-Cas9 ribonucleoproteins. Nat Biotech. 2015;33(11):1162-4.

20. Baltes NJ, et al. DNA replicons for plant genome engineering. Plant Cell. 2014;26(1):151-63.

21. Demirer GS, et al. High aspect ratio nanomaterials enable delivery of functional genetic material without DNA integration in mature plants. Nat Nanotechnol. 2019;14(5):456-64.

22. Kwak SY, et al. Chloroplast-selective gene delivery and expression in planta using chitosan-complexed single-walled carbon nanotube carriers. Nat Nanotechnol. 2019;14(5):447-55.

23. Waltz E. Gene-edited CRISPR mushroom escapes US regulation. Nature. 2016;532(7599):293.

24. Waltz E. CRISPR-edited crops free to enter market, skip regulation. Nat Biotechnol. 2016;34(6):582.

25. Waltz E. With a free pass, CRISPR-edited plants reach market in record time. Nat Biotechnol. 2018;36(1):6-7.

26. Pickar-Oliver A, Gersbach CA. The next generation of CRISPR-Cas technologies and applications. Nat Rev Mol Cell Biol. 2019;20(8):490-507.

27. Stella S, Montoya G. The genome editing revolution: a CRISPR-Cas TALE off-target story. Bioessays. 2016;38(S1):S4-S13. 
28. Chen K, et al. CRISPR/Cas genome editing and precision plant breeding in agriculture. Annu Rev Plant Biol. 2019; 70:667-97.

29. Li JF, et al. Multiplex and homologous recombination-mediated genome editing in Arabidopsis and Nicotiana benthamiana using guide RNA and Cas9. Nat Biotechnol. 2013;31(8):688-91.

30. Shan Q, et al. Targeted genome modification of crop plants using a CRISPR-Cas system. Nat Biotechnol. 2013; 31(8):686-8.

31. Nekrasov V, et al. Targeted mutagenesis in the model plant Nicotiana benthamiana using Cas9 RNA-guided endonuclease. Nat Biotechnol. 2013;31(8):691-3.

32. Hanna RE, Doench JG. Design and analysis of CRISPR-Cas experiments. Nat Biotechnol. 2020;38(7):813-23.

33. Jinek $M$, et al. A programmable dual-RNA-guided DNA endonuclease in adaptive bacterial immunity. Science. 2012; 337(6096):816-21.

34. Wright AV, Nunez JK, Doudna JA. Biology and applications of CRISPR systems: harnessing nature's toolbox for genome engineering. Cell. 2016;164(1-2):29-44.

35. O'Connell MR, et al. Programmable RNA recognition and cleavage by CRISPR/Cas9. Nature. 2014;516(7530):263-6.

36. Zaidi SS, Mahfouz MM, Mansoor S. CRISPR-Cpf1: a new tool for plant genome editing. Trends Plant Sci. 2017;22(7):550-3.

37. Aman R, et al. RNA virus interference via CRISPR/Cas13a system in plants. Genome Biol. 2018;19(1):1.

38. Abudayyeh 00 , et al. RNA targeting with CRISPR-Cas13. Nature. 2017; 550:280-84.

39. Smargon AA, et al. Cas $13 \mathrm{~b}$ is a type VI-B CRISPR-associated RNA-guided RNase differentially regulated by accessory proteins Csx27 and Csx28. Mol Cell. 2017;65(4):618-30 e7.

40. Guilinger JP, Thompson DB, Liu DR. Fusion of catalytically inactive Cas9 to Fokl nuclease improves the specificity of genome modification. Nat Biotechnol. 2014;32(6):577-82.

41. Li, Z., et al., A potent Cas9-derived gene activator for plant and mammalian cells. Nat Plants. 2017:3:930-36.

42. Piatek $A$, et al. RNA-guided transcriptional regulation in planta via synthetic dCas9-based transcription factors. Plant Biotechnol J. 2015;13(4):578-89.

43. Terns MP. CRISPR-based technologies: impact of RNA-targeting systems. Mol Cell. 2018;72(3):404-12.

44. Shimatani, Z., et al., Targeted base editing in rice and tomato using a CRISPR-Cas9 cytidine deaminase fusion. Nat Biotechnol. 2017;35:441-43.

45. Zaidi SS, Mukhtar MS, Mansoor S. Genome editing: targeting susceptibility genes for plant disease resistance. Trends Biotechnol. 2018;36(9):898-906.

46. Chandrasekaran J, et al. Development of broad virus resistance in non-transgenic cucumber using CRISPR/Cas9 technology. Mol Plant Pathol. 2016;17(7):1140-53.

47. Pyott DE, Sheehan E, Molnar A. Engineering of CRISPR/Cas9-mediated potyvirus resistance in transgene-free Arabidopsis plants. Mol Plant Pathol. 2016;17(8):1276-88.

48. Blanvillain-Baufume $\mathrm{S}$, et al. Targeted promoter editing for rice resistance to Xanthomonas oryzae pv. oryzae reveals differential activities for SWEET14-inducing TAL effectors. Plant Biotechnol J. 2017:15(3):306-17.

49. Li T, et al. High-efficiency TALEN-based gene editing produces disease-resistant rice. Nat Biotech. 2012;30(5):390-2.

50. $\mathrm{Xu} \mathrm{Z}$, et al. Engineering broad-spectrum bacterial blight resistance by simultaneously disrupting variable TALE-binding elements of multiple susceptibility genes in rice. Mol Plant. 2019;12(11):1434-46.

51. Oliva R, et al. Broad-spectrum resistance to bacterial blight in rice using genome editing. Nat Biotechnol. 2019;37(11): 1344-50.

52. Si $X$, et al. Manipulating gene translation in plants by CRISPR-Cas9-mediated genome editing of upstream open reading frames. Nat Protoc. 2020:15(2):338-63.

53. Cai Y, et al. CRISPR/Cas9-mediated deletion of large genomic fragments in soybean. Int J Mol Sci. 2018;19(12):3835

54. Zhou H, et al. Large chromosomal deletions and heritable small genetic changes induced by CRISPR/Cas9 in rice. Nucleic Acids Res. 2014;42(17):10903-14.

55. Nishimasu H, et al. Engineered CRISPR-Cas9 nuclease with expanded targeting space. Science. 2018;361(6408):1259-62.

56. Ashfield T, et al. Evolution of a complex disease resistance gene cluster in diploid Phaseolus and tetraploid Glycine. Plant Physiol. 2012;159(1):336-54.

57. Van Vu, T., et al., Challenges and perspectives in homology-directed gene targeting in monocot plants. Rice. 2019;12:95.

58. Liu M, et al. Methodologies for improving HDR efficiency. Front Genet. 2019;9:691.

59. Butt $\mathrm{H}$, et al. Efficient CRISPR/Cas9-mediated genome editing using a chimeric single-guide RNA molecule. Front Plant Sci. 2017;8:1441.

60. Wang $\mathrm{M}$, et al. Gene targeting by homology-directed repair in rice using a geminivirus-based CRISPR/Cas9 system. Mol Plant. 2017;10(7):1007-10.

61. Zhang $\mathrm{H}$, et al. Back into the wild-apply untapped genetic diversity of wild relatives for crop improvement. Evol Appl. 2017;10(1):5-24.

62. Arora $\mathrm{S}$, et al. Resistance gene cloning from a wild crop relative by sequence capture and association genetics. Nat Biotechnol. 2019;37(2):139-43.

63. Kourelis J, van der Hoorn RAL. Defended to the nines: 25 years of resistance gene cloning identifies nine mechanisms for R protein function. Plant Cell. 2018:30(2);285-99.

64. Komor AC, et al. Programmable editing of a target base in genomic DNA without double-stranded DNA cleavage. Nature. 2016;533(7603):420-4.

65. Bastet $A$, et al. Mimicking natural polymorphism in elF4E by CRISPR-Cas9 base editing is associated with resistance to potyviruses. Plant Biotechnol J. 2019;17(9):1736-50.

66. Butt $\mathrm{H}$, et al. CRISPR directed evolution of the spliceosome for resistance to splicing inhibitors. Genome Biol. 2019;20(1):73.

67. Li C, et al. Targeted, random mutagenesis of plant genes with dual cytosine and adenine base editors. Nat Biotechnol. 2020;38(7):875-82.

68. Butt H, et al. CRISPR-based directed evolution for crop improvement. Trends Biotechnol. 2020;38(3):236-40.

69. Aman, R., et al., Engineering RNA virus interference via the CRISPR/Cas13 machinery in Arabidopsis. Viruses. 2018;10(12):732.

70. Zhang T, et al. Establishing CRISPR/Cas13a immune system conferring RNA virus resistance in both dicot and monocot plants. Plant Biotechnol J. 2019;17(7):1185-7. 
71. Zhang T, et al. Establishing RNA virus resistance in plants by harnessing CRISPR immune system. Plant Biotechnol J. 2018;16(8):1415-23.

72. Ali Z, et al. CRISPR/Cas9-mediated viral interference in plants. Genome Biol. 2015;16(1):238.

73. Ali Z, et al. CRISPR/Cas9-mediated immunity to geminiviruses: differential interference and evasion. Sci Rep. 2016;6: 26912.

74. Baltes NJ, et al. Conferring resistance to geminiviruses with the CRISPR-Cas prokaryotic immune system. Nat Plants. 2015;1(10):15145

75. Ji X, et al. Establishing a CRISPR-Cas-like immune system conferring DNA virus resistance in plants. Nat Plants. 2015;1 (10): 15144.

76. Tashkandi M, et al. Engineering resistance against Tomato yellow leaf curl virus via the CRISPR/Cas9 system in tomato. Plant Signal Behav. 2018;13(10):e1525996.

77. Yin, K., et al., Engineer complete resistance to Cotton Leaf Curl Multan virus by the CRISPR/Cas9 system in Nicotiana benthamiana. Phytopathol Res. 2019;1:9.

78. Mehta $\mathrm{D}$, et al. Linking CRISPR-Cas9 interference in cassava to the evolution of editing-resistant geminiviruses. Genome Biol. 2019:20(1):80.

79. Roy A, et al. Multiplexed editing of a begomovirus genome restricts escape mutant formation and disease development. Plos One. 2019;14(10):e0223765.

80. Tripathi JN, et al. CRISPR/Cas9 editing of endogenous banana streak virus in the B genome of Musa spp. overcomes a major challenge in banana breeding. Commun Biol. 2019;2:46.

81. Macovei A, et al. Novel alleles of rice elF4G generated by CRISPR/Cas9-targeted mutagenesis confer resistance to Rice tungro spherical virus. Plant Biotechnol J. 2018;16(11):1918-27.

82. Gomez MA, et al. Simultaneous CRISPR/Cas9-mediated editing of cassava elF4E isoforms nCBP-1 and nCBP-2 reduces cassava brown streak disease symptom severity and incidence. Plant Biotechnol J. 2019;17(2):421-34.

83. Santillan Martinez MI, et al. CRISPR/Cas9-targeted mutagenesis of the tomato susceptibility gene PMR4 for resistance against powdery mildew. BMC Plant Biol. 2020;20(1):284.

84. Zhang S, et al. Knockout of SIMAPK3 reduced disease resistance to Botrytis cinerea in tomato plants. J Agric Food Chem. 2018;66(34):8949-56.

85. Prihatna C, Barbetti MJ, Barker SJ. A novel tomato fusarium wilt tolerance gene. Front Microbiol. 2018:9:1226

86. Wang F, et al. Enhanced rice blast resistance by CRISPR/Cas9-targeted mutagenesis of the ERF transcription factor gene OsERF922. PLoS One. 2016;11(4):e0154027.

87. Wang X, et al. CRISPR/Cas9-mediated efficient targeted mutagenesis in grape in the first generation. Plant Biotechnol J. 2017:16(4);844-55.

88. Nekrasov V, et al. Rapid generation of a transgene-free powdery mildew resistant tomato by genome deletion. Sci Rep. 2017;7(1):482.

89. Dale J, et al. Transgenic Cavendish bananas with resistance to Fusarium wilt tropical race 4. Nat Commun. 2017:8(1):1496.

90. Xie K, Yang Y. RNA-guided genome editing in plants using a CRISPR-Cas system. Mol Plant. 2013;6(6):1975-83.

91. Malnoy M, et al. DNA-free genetically edited grapevine and apple protoplast using CRISPR/Cas9 ribonucleoproteins. Front Plant Sci. 2016;7(1904):1904.

92. Wang $Y$, et al. Simultaneous editing of three homoeoalleles in hexaploid bread wheat confers heritable resistance to powdery mildew. Nat Biotechnol. 2014;32(9):947-51.

93. Zhang Y, et al. Simultaneous modification of three homoeologs of TaEDR1 by genome editing enhances powdery mildew resistance in wheat. Plant J. 2017:91(4):714-24.

94. Zhou J, et al. Gene targeting by the TAL effector PthXo2 reveals cryptic resistance gene for bacterial blight of rice. Plant J. 2015;82(4):632-43.

95. Kim YA, Moon H, Park CJ. CRISPR/Cas9-targeted mutagenesis of Os8N3 in rice to confer resistance to Xanthomonas oryzae pv. oryzae. Rice (N Y). 2019;12(1):67.

96. Jiang W, et al. Demonstration of CRISPR/Cas9/sgRNA-mediated targeted gene modification in Arabidopsis, tobacco, sorghum and rice. Nucleic Acids Res. 2013:41(20):e188.

97. Ortigosa A, et al. Design of a bacterial speck resistant tomato by CRISPR/Cas9-mediated editing of SIJAZ2. Plant Biotechnol J. 2019;17(3):665-73.

98. de Toledo Thomazella, D.P., et al., CRISPR-Cas9 mediated mutagenesis of a DMR6 ortholog in tomato confers broadspectrum disease resistance. BioRxiv, 2016: p. doi: https://doi.org/10.1101/064824.

99. Jia $\mathrm{H}$, et al. Genome editing of the disease susceptibility gene CsLOB1 in citrus confers resistance to citrus canker. Plant Biotechnol J. 2017;15(7):817-23.

100. Peng A, et al. Engineering canker-resistant plants through CRISPR/Cas9-targeted editing of the susceptibility gene CsLOB1 promoter in citrus. Plant Biotechnol J. 2017;15(12):1509-19.

101. Gumtow R, et al. A Phytophthora palmivora extracellular cystatin-like protease inhibitor targets papain to contribute to virulence on papaya. Mol Plant-Microbe Interact. 2018;31(3):363-73.

102. Fister AS, et al. Transient expression of CRISPR/Cas9 machinery targeting TcNPR3 enhances defense response in Theobroma cacao. Front Plant Sci. 2018;9:268.

103. Zaidi SS, et al. Engineering plants for geminivirus resistance with CRISPR/Cas9 system. Trends Plant Sci. 2016;21 (4):279-81

104. Mahas A, Aman R, Mahfouz M. CRISPR-Cas13d mediates robust RNA virus interference in plants. Genome Biol. 2019; 20(1):263.

105. Hadidi A. Next-generation sequencing and CRISPR/Cas13 editing in viroid research and molecular diagnostics. Viruses. 2019;11(2):120

106. Rybicki EP. CRISPR-Cas9 strikes out in cassava. Nat Biotechnol. 2019;37(7):727-8.

107. Zaidi SS, et al. Engineering plant immunity: using CRISPR/Cas9 to generate virus resistance. Front Plant Sci. 2016;7:1673.

108. Giraud T, Gladieux P, Gavrilets S. Linking the emergence of fungal plant diseases with ecological speciation. Trends Ecol Evol. 2010;25(7):387-95.

109. Acevedo-Garcia J, Kusch S, Panstruga R. Magical mystery tour: MLO proteins in plant immunity and beyond. New Phytol. 2014;204(2):273-81. 
110. Freisleben $R$, Lein A. Über die Auffindung einer mehltauresistenten Mutante nach Röntgenbestrahlung einer anfälligen reinen Linie von Sommergerste. Naturwissenschaften. 1942;30(40):608.

111. Jørgensen IH. Discovery, characterization and exploitation of Mlo powdery mildew resistance in barley. Euphytica. 1992; 63(1-2):141-52.

112. van Schie CC, Takken FL. Susceptibility genes 101: how to be a good host. Annu Rev Phytopathol. 2014;52:551-81.

113. Maher MF, et al. Plant gene editing through de novo induction of meristems. Nat Biotechnol. 2020;38(1):84-9.

114. Merker $L$, et al. Enhancing in planta gene targeting efficiencies in Arabidopsis using temperature-tolerant CRISPR/ LbCas12a. Plant Biotechnol J. 2020;18:2382-84.

115. Lu Y, Tian Y, Shen R. et al. Targeted, efficient sequence insertion and replacement in rice. Nat Biotechnol. 2020. https:/ doi.org/10.1038/s41587-020-0581-5.

116. Barone $P$, et al. Efficient gene targeting in maize using inducible CRISPR-Cas9 and marker-free donor template. Mol Plant. 2020;13(8):1219-27.

117. Beying N, et al. CRISPR-Cas9-mediated induction of heritable chromosomal translocations in Arabidopsis. Nat Plants. 2020;6(6):638-45.

118. Schmidt C, et al. Changing local recombination patterns in Arabidopsis by CRISPR/Cas mediated chromosome engineering. Nat Commun. 2020;11(1):4418.

119. Hanley-Bowdoin $\mathrm{L}$, et al. Geminiviruses: masters at redirecting and reprogramming plant processes. Nat Rev Microbiol. 2013;11(11):777-88

120. Yang X, et al. Geminivirus-associated betasatellites: exploiting chinks in the antiviral arsenal of plants. Trends Plant Sci. 2019;24(6):519-29.

121. Zubair $\mathrm{M}$, et al. An insight into Cotton leaf curl Multan betasatellite, the most important component of cotton leaf cur disease complex. Viruses. 2017:9(10):280.

122. Aguilar E, Garnelo Gomez B, Lozano-Duran R. Recent advances on the plant manipulation by geminiviruses. Curr Opin Plant Biol. 2020;56:56-64.

123. Custers, R., The regulatory status of gene-edited agricultural products in the EU and beyond. Emerg Top Life Sci. 2017; 1(2):221-29.

124. Globus R, Qimron U. A technological and regulatory outlook on CRISPR crop editing. J Cell Biochem. 2017;119:1291-98.

125. Huang $S$, et al. A proposed regulatory framework for genome-edited crops. Nat Genet. 2016;48(2):109-11.

126. Eriksson D, et al. Why the European Union needs a national GMO opt-in mechanism. Nat Biotechnol. 2018;36(1):18-9.

127. Ali, Z., et al., Fusion of the Cas9 endonuclease and the VirD2 relaxase facilitates homology-directed repair for precise genome engineering in rice. Commun Biol. 2020;3:44.

\section{Publisher's Note}

Springer Nature remains neutral with regard to jurisdictional claims in published maps and institutional affiliations.

\section{Ready to submit your research? Choose BMC and benefit from:}

- fast, convenient online submission

- thorough peer review by experienced researchers in your field

- rapid publication on acceptance

- support for research data, including large and complex data types

- gold Open Access which fosters wider collaboration and increased citations

- maximum visibility for your research: over $100 \mathrm{M}$ website views per year

At $\mathrm{BMC}$, research is always in progress. 